\title{
Postural ergonomics during robotic and laparoscopic gastric bypass surgery: a pilot project
}

\author{
Elise H. Lawson • Myriam J. Curet • Barry R. Sanchez • \\ Rob Schuster • Ramon Berguer
}

Received: 17 November 2006 / Accepted: 15 January 2007 / Published online: 10 February 2007

C) Springer London 2007

\begin{abstract}
We hypothesized that a laparoscopic technique for Roux-en-Y gastric bypass surgery is associated with more musculoskeletal discomfort and ergonomic strain than a robotic technique. This pilot project studied one surgeon while he performed four laparoscopic and four robotic (da Vinci system) Rouxen-Y gastric bypass procedures. We measured musculoskeletal discomfort with body part discomfort score (BPD) and ergonomic positioning with the rapid upper-limb assessment tool (RULA). At the end of the case, the robotic cases were associated with more discomfort in the neck (median BPD scores 2.5 versus 1.0, $P=0.028$ ), while the laparoscopic cases were associated with more discomfort in the upper back (median BPD scores 2.0 versus 1.0, $P=0.028$ ). Both the right and left shoulders demonstrated more discomfort with the laparoscopic group (median BPD scores 3.0 versus $1.5, P=0.057)$. The RULA analysis demonstrated that the upper arm (1.0 versus 2.25), lower arm (1.125 versus 2.125$)$, wrist (2.5 versus 3.5 ) and wrist twist (1.25 versus 2 ) were held in less ergonomically correct
\end{abstract}

\author{
E. H. Lawson · M. J. Curet $(\bowtie) \cdot$ R. Schuster \\ Department of Surgery, H3680, Stanford University, \\ 300 Pasteur Dr, Stanford, CA 94305, USA \\ e-mail: mcuret@stanford.edu \\ B. R. Sanchez \\ Peninsula Surgical Specialists Medical Group, \\ Burlingame, CA, USA \\ R. Berguer \\ Department of Surgery, University of California Davis, \\ Davis, CA, USA \\ R. Berguer \\ Department of Surgery, \\ Contra Costa Regional Medical Center, Davis, CA, USA
}

positioning (higher score) in the laparoscopic group compared to the robotic group $(P=0.029)$. In contrast, the trunk (1.5 versus 1.0$)$ had a worse RULA score in the robotic group compared to the laparoscopic cases. These pilot data suggest that robotic Roux-en-Y gastric bypass surgery may result in less musculoskeletal stress to the upper extremities than standard laparoscopic technique. In contrast, robotic surgery seems to offer both postural advantages and disadvantages for the neck and back region. More-detailed studies are needed to fully assess the potential postural advantages of robotic surgical techniques over standard laparoscopy.

Keywords Ergonomics - Gastric bypass surgery · Laparoscopy $\cdot$ Robotic surgery

\section{Introduction}

Obesity is a complex disease that has become a major cause of morbidity and mortality in the United States. Roux-en-Y gastric bypass (RYGBP) surgery has emerged as the weight-loss surgery of choice for patients with clinical severe obesity, generally defined as a body mass index (BMI) of $\geq 40 \mathrm{~kg} / \mathrm{m}^{2}$ or $\geq 35 \mathrm{~kg} / \mathrm{m}^{2}$ with two or more co-morbid conditions. The number of surgeries performed has increased in recent years as it proves to be a relatively safe and cost-effective treatment modality for achieving significant, sustained weight loss [1, 2]. RYGBP surgery has further proven to be effective at reducing common co-morbidities of obesity including type 2 diabetes mellitus, hypertension, and dyslipidemia $[2,3]$.

The laparoscopic technique for RYGBP surgery has become the dominant method, though select patients 
still undergo open surgery $[1,4]$. Laparoscopic RYGBP is a technically challenging operation with significant ergonomic strain on the surgeon. In general, laparoscopic surgery places strain on the neck, trunk, and upper extremities by requiring the operator to assume awkward, non-ergonomic positions [5-7]. Laparoscopic surgery has further proven to be more physically demanding $[6,8]$ and mentally stressful [9] to the operator then performing open surgery. Numerous reasons have been cited to explain this increased demand on the surgeon, including the static head and trunk position required for laparoscopic surgery $[7,10]$ and the use of long, fulcrumed laparoscopic instruments that require increased movement of the shoulders and upper arms [11]. These explanations combined with the awkward design and poor mechanical efficiency of laparoscopic instruments lead to increased musculoskeletal complaints and fatigue, as well as the possibility of work-related musculoskeletal injury $[5,12,13]$.

A totally robotic laparoscopic RYGBP procedure using the da Vinci surgical system (Intuitive Surgical Inc., Sunnyvale, CA, USA) has recently been described as a safe and effective alternative to open or laparoscopic techniques [14]. The robot is a telemanipulator instrument that allows the surgeon to control up to three robotic arms with surgical instruments from a remote console. Studies have shown equal performance in terms of precision and efficiency between laparoscopic and robotic surgery [15], as well as similar rates of mortality, complications and length of stay [16]. Initial reports on robotic surgery also suggest that it is more ergonomically favorable and possibly less mentally stressful than traditional laparoscopic surgery $[14,16,17]$. This pilot study compared the musculoskeletal discomfort and postural ergonomics of laparoscopic and robotic techniques for RYGBP surgery in a real-life clinical setting with the goal of elucidating the advantages and drawbacks of each technique. The hypothesis was that a laparoscopic technique for Rouxen-Y gastric bypass surgery is associated with more musculoskeletal discomfort and ergonomic strain than a robotic technique.

\section{Methods}

This is a pilot study conducted at the Stanford University Medical Center. One minimally invasive surgery (MIS) fellow, who had already performed 20 robotic and 20 laparoscopic RYGBP cases was studied while performing a series of four laparoscopic and four robotic RYGBP procedures in the operating room. The robotic surgeries were performed using the da
Vinci surgical system. The technique has been previously described [14]. For both the laparoscopic and robotic cases, the jejunojejunostomy was created with a linear stapler and the resulting enterotomy handsewn while the gastrojejunostomy was hand-sewn in two layers.

At the beginning, middle (after jejunojejunostomy) and end of each case, the MIS fellow completed a body part discomfort (BPD) questionnaire by hand (the surgeon broke scrub in the middle of the case to complete the questionnaire). On the questionnaire, the MIS fellow rated the amount of musculoskeletal discomfort in his neck, shoulders, upper and lower back, hands, wrists, and buttocks on a drawing of a human body. A five-point scale was used, with 1 being minimal discomfort, 3 moderate discomfort and 5 severe discomfort.

The surgeon was also videotaped from the side while performing the surgeries, providing a view from the head to upper legs. Following the surgery, the videotapes of each operation were analyzed and scored by two independent observers. The position of the surgeon's upper arm, lower arm, wrist, neck and trunk were scored using the rapid upper-limb assessment (RULA) tool at various points throughout the surgery. Ergonomically correct was defined for the RULA as having the spine upright, the elbows at the side bent at $90^{\circ}$ with no bend or twist at the wrists. Scores were assigned based on how far from ergonomically correct the surgeon was positioned. Data points were taken during the steps of the surgery where the surgeon measures the Roux limb (Step 1), during creation of the jejunojejunostomy (Step 2) and during creation of the gastrojejunostomy (Step 3) which was hand-sewn in two layers. The scores for the two observers were averaged for each time data point.

Following data collection, the Mann-Whitney $U$ or analysis of variations (ANOVA) chi-square test was used for statistical analysis, with a significant $P$ value defined as less than 0.05 .

\section{Results}

Laparoscopic RYGBP was performed on four patients and robotic RYGBP was performed on four patients by an MIS fellow who had completed 9 months of his 12 month fellowship. All eight patients were female. Mean age for the laparoscopic group (54.3 years; range 39-60) was higher than for the robotic group (36.8 years, range $28-46 ; P=0.038$ ) but there was no significant difference in mean pre-operative BMI (laparoscopic: $44.3 \mathrm{~kg} / \mathrm{m}^{2}$, range $37.5-55.6$; robotic: 
$43.7 \mathrm{~kg} / \mathrm{m}^{2}$, range $\left.37.1-56.6\right)$. Operative times were shorter for the robotic patients (105 min; range 90-120) than for the laparoscopic cases (133.8 min; range 120-155; $P=0.030)$.

The surgeon experienced worsening musculoskeletal discomfort from beginning to end for both the laparoscopic and robotic groups. Median body part discomfort scores (BPD) for the laparoscopic and robotic cases are reported in Fig. 1. At the beginning of each case, the surgeon reported minimal musculoskeletal discomfort. The data from the mid-point of the cases (after creation of the jejunojejunostomy) demonstrate a trend towards greater discomfort in the shoul-
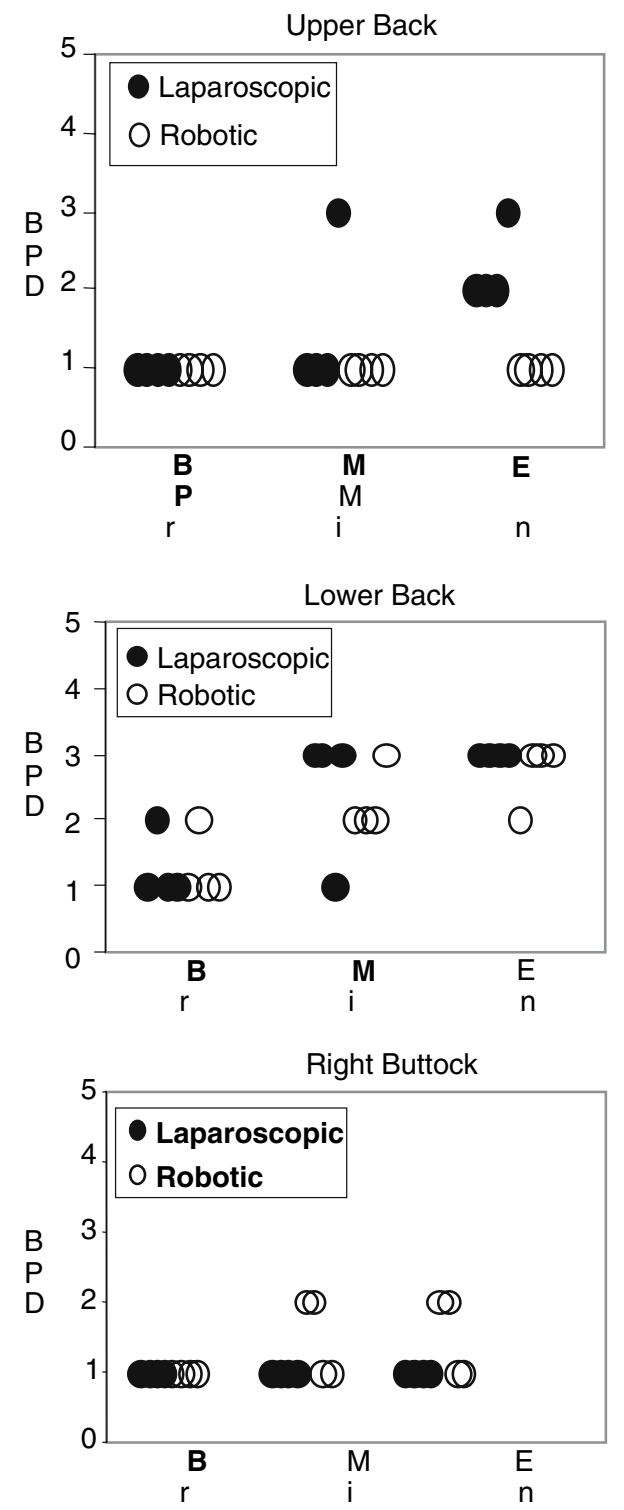

Fig. 1 Body part discomfort scores for laparoscopic and robotic cases. $B P D$ body part discomfort, $B$ beginning of operation, $M$ middle of operation (after jejunojejunostomy), $E$ end of operation ders, wrists, hands and lower back for the laparoscopic cases and in the neck and buttocks for the robotic cases; however, these differences do not reach statistical significance. At the end of the case, the robotic
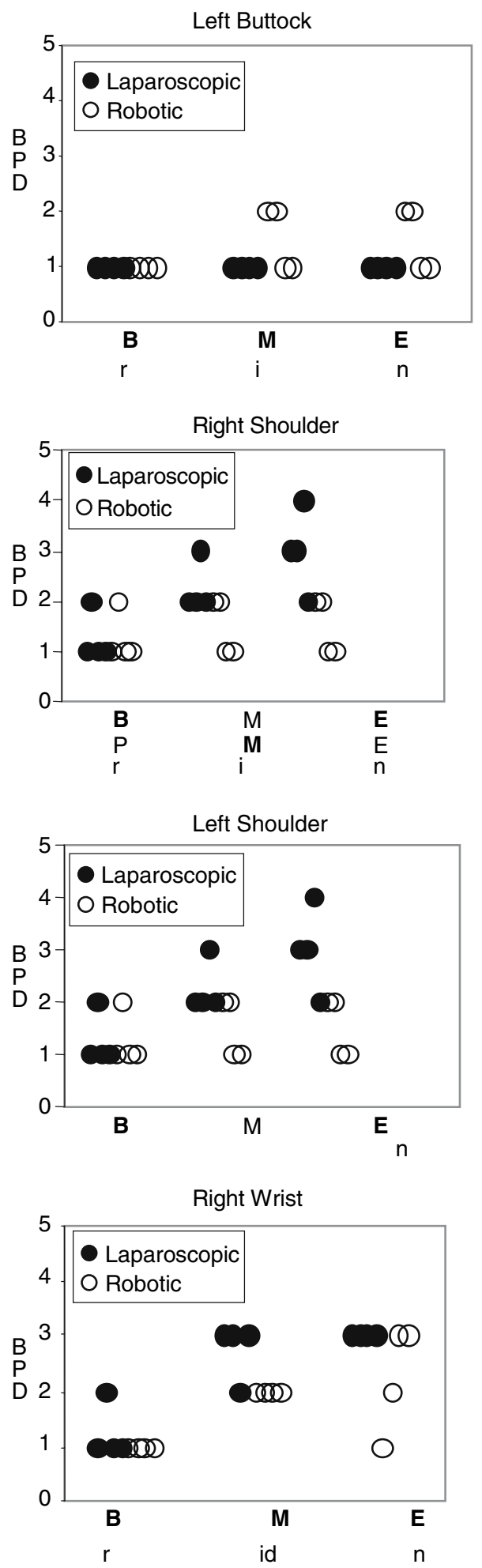

Fig. 1 continued 

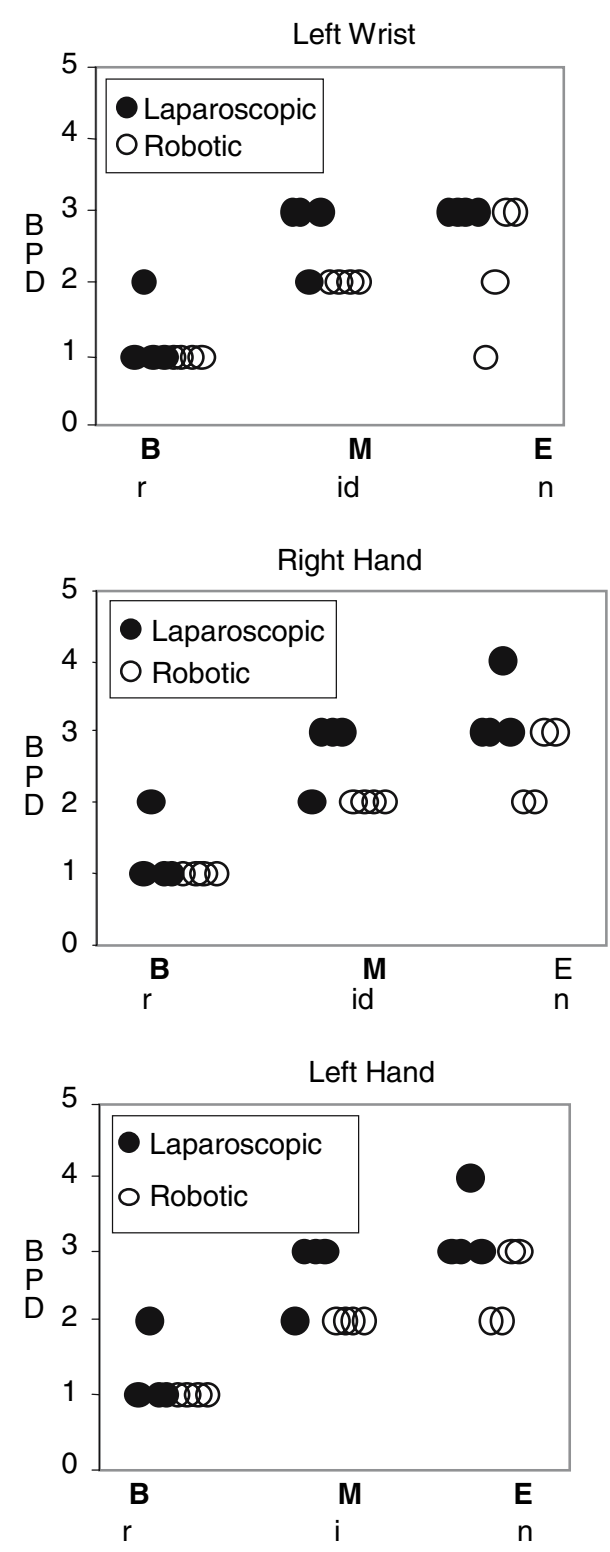

BPD $=$ Body Part Discomfort

$\mathrm{B}=$ beginning of operation

$\mathrm{M}=$ middle of operation ( after jejunojejunostomy)

$\mathrm{E}=\mathrm{end}$ of operation

Fig. 1 continued

cases were associated with more discomfort in the neck (median BPD scores 2.5 versus 1.0, $P=0.028$ ), while the laparoscopic cases were associated with more discomfort in the upper back (median BPD scores 2.0 versus $1.0, P=0.028$ ). Both the right and left shoulders demonstrated more discomfort with the laparoscopic group but the differences did not reach statistical significance (median BPD scores 3.0 versus 1.5, $P=0.057)$.
Results of the RULA analysis were not significantly different for the three steps during the surgery. Data from the Step 3 RULA analysis (creation of the gastrojejunostomy-Fig. 2) demonstrated that the upper arm, lower arm, wrist position, and wrist twist were held in less ergonomically correct positioning in the laparoscopic group compared to the robotic group ( 2.25 versus $1.0,2.125$ versus $1.125,3.5$ versus 2.5 , and 2 versus 1.25 , respectively; $P=0.029$ ). In contrast, the trunk had a lower RULA score in the laparoscopic group compared to the robotic group (1.0 versus 1.5; $P=0.029)$.
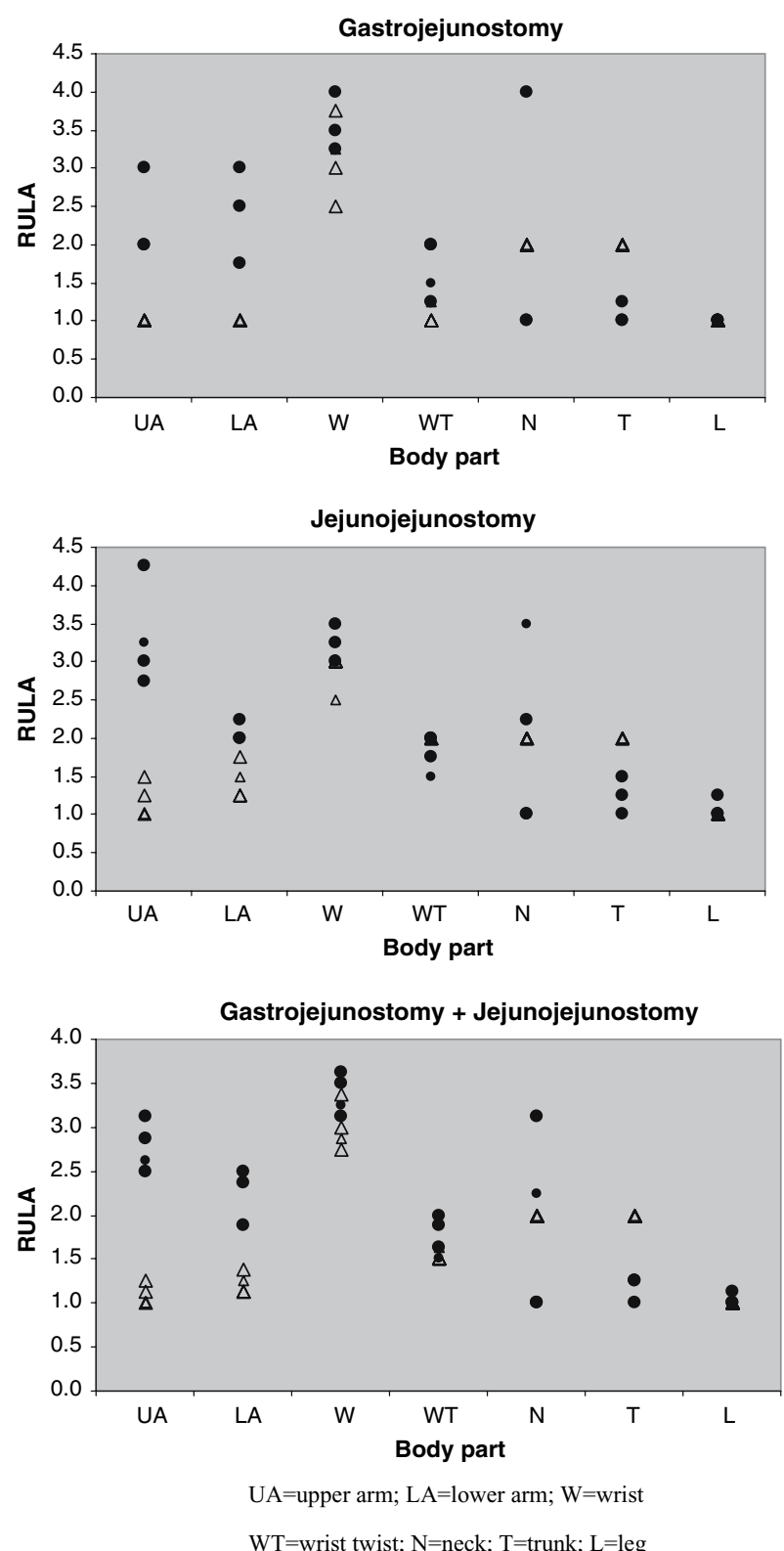

Fig. 2 RULA scores for Steps 1 and 2 for laparoscopic and robotic cases. $U A$ upper arm; $L A$ lower arm; $W$ wrist; $W T$ wrist twist; $N$ neck; $T$ trunk; $L$ leg 


\section{Discussion}

RYGBP is a technically and ergonomically challenging surgery to perform using the laparoscopic technique. While laparoscopic surgery frequently results in improved recovery time and reduced hospital length of stay for patients compared to open surgery, it often does so at the expense of the surgeon's effort. Multiple studies have documented the ergonomic problems associated with laparoscopic surgery $[5-7,10,12,13$, 17]. Laparoscopic surgery has been found to necessitate relatively high muscular loading compared to open surgery, and also requires the surgeon to assume atypical, awkward postures for extended periods of time [8, 13]. The upper extremities perform repetitive awkward movements while the neck and trunk are maintained in a more static posture, both leading to physical stress on the surgeon. [7] Questionnaire-based studies on laparoscopic surgeons have found increased rates of finger numbness and eye strain [12] as well as reports of frequent pain or numbness in the neck and upper extremities following laparoscopic operations [6].

The da Vinci telerobotic surgical system was developed in response to some of the major challenges associated with traditional laparoscopic techniques [18], [19]. Technical limitations of laparoscopy, including the fulcrum effect caused by the body wall and the long length of laparoscopic instruments, are avoided by the master/slave design in which the surgeon sits at a remote console and controls up to three robotic arms with surgical instruments. Dexterity and agility are also improved while physiologic tremor and friction are reduced by electronically controlling the seven-degreeof-freedom instruments [18]. Surgeons report that this feature results in improved tissue manipulation and specifically is better for fine tissue dissection and suturing then traditional laparoscopic instruments [16]. The robot is further designed with a binocular endoscope and a stable camera platform, providing the surgeon with a steady three-dimensional view of the surgical field, in contrast to the unstable hand-held camera and flat two-dimensional field seen with traditional laparoscopy $[18,20]$.

The da Vinci robot was initially designed for cardiac surgery and has been used extensively by cardiac surgeons for procedures such as coronary artery bypass grafting and repair of mitral valves and atrial septal defects [20, 21]. Multiple abdominal surgeries can also be safely performed with the robot, including cholecystectomy, esophagectomy, fundoplication, Heller myotomy, gastrectomy, splenectomy, pancreatectomy, collectomy and RYGBP. Urologic and gynecologic surgeries have also been described [20,22].
Though the da Vinci robot was designed with an ergonomically optimal operating position in mind, relatively few studies have investigated this topic. For example, robotic surgery may mitigate the excessive flexion and ulnar deviation at the wrist seen with the use of traditional laparoscopic instruments because the surgeon is able to control the movement of the instruments from a remote console while a seven-degree-offreedom actuator moves the instruments. Thus the robotic system more closely mimics the movement of the human wrist and hand while physically assuming the awkward positions and forces that would otherwise be put directly on the surgeon. A recently published study comparing robotic and laparoscopic technique for completing a set of simulated surgical tasks in the laboratory setting reported significantly lower RULA and job strain index (JSI) scores for the robot, leading the authors to conclude that robotic surgery does not add additional mental stress to the surgeon and provides a more comfortable environment in which to work [17]. The authors evaluated ergonomic results in medical students, junior internal medicine and junior surgery residents who had no laparoscopic experience in the laboratory with simulated surgical tasks. Our study evaluated a board-eligible surgeon who had completed his entire general surgical residency with advanced laparoscopic skills in a clinical setting during an actual operation. In this situation, we were assessing real-life ergonomic problems. Although this is clearly a pilot study, our results are consistent with the published literature, indicating that robotic RYGBP surgery is associated with less musculoskeletal discomfort in the upper back and more-ergonomic positioning of the upper arm, lower arm, wrist, and wrist twist.

The improvement in ergonomics and comfort of robotic surgery do not seem to have an adverse effect on performance compared to laparoscopic surgery [15]. Our data demonstrated that operative times were significantly shorter for the robotic cases than for the laparoscopic cases. This is consistent with the first description of totally robotic RYGBP surgery, which reported comparable operating times with the laparoscopic technique, no change in the rate of minor and major complications and a very short learning curve [14]. A study evaluating the learning curve for suturing and dexterity skills using either the da Vinci robot or standard laparoscopy showed that novice surgeons learn difficult tasks more quickly using the robot [23]. Another prospective analysis of a variety of robotassisted surgical procedures (211 in total) reported comparable results compared to traditional laparoscopic surgery in terms of mortality, complications and length of stay [16]. A further study on aortic replacement 
in pigs found the robot-assisted technique to be superior to standard laparoscopic technique due to shorter operating time, fewer complications and less blood loss [24].

Other studies, however, report time loss with robotassisted laparoscopic cholecystectomy compared to traditional techniques, with one study citing increased time required for set up and sterile draping [25] and another citing increased operation time due to slow, cautious movement of the robot arms by the surgeon and inappropriate instruments [26]. Another study comparing laparoscopic skills performance between standard instruments and the da Vinci robotic system found no time advantage when performing fine tasks (intracorporeal knot tying and running stitches with 4-0, 6-0 and 7-0 sutures), though the robot was more precise. They further determined that basic task performance (running a $100-\mathrm{cm}$ rope, placing beads onto pins, and dropping peanuts into cylinders) with standard instruments was actually faster and equally precise as with the robotic system. The authors thus conclude that robotic systems are not of benefit for general surgical procedures, but may be more useful in surgeries that require more fine suturing [27]. A study comparing robotic with standard laparoscopic technique for performing a small-bowel anastomosis in a porcine experimental setup showed significantly shorter time required per stitch for the robot and more stitch errors for the standard group. These authors thus concluded that robotic assistance may be of greater benefit when performing more-complex manipulative maneuvers [28].

Haptic sensors have not yet been incorporated into the da Vinci robot, thus visual cues must be used to determine tactile and tensile forces. This lack of tactile feedback is reported as a deficit of the system, especially in determining tissue and suture tension [16, 20, $26,28]$. Another potential drawback to robotic surgery is the effect of the work station and the viewing angle on discomfort in the neck. The surgeon sits at a console and looks down into the visual field throughout the case. The higher neck BPD and trunk RULA scores seen at the end of the robotic cases may reflect an ergonomic disadvantage to the current workstation of the da Vinci system.

\section{Limitations}

This article reports a series of pilot observations on one subject and further study in a large sample size is needed to validate the observations. The surgeon's posture was only evaluated from one side due to the set up of the video camera, thus the results should be considered approximate, rather than absolute posture assessments. The RULA methodology has been used in previous ergonomic studies and has undergone validation for assessing the postures of the neck, trunk and upper limbs [18]. The possibility of introducing random error while scoring the videos was reduced by having two observers score the tapes independently.

\section{Conclusions}

In this pilot study, we assessed the musculoskeletal stress and ergonomic positioning associated with robotic and laparoscopic RYGBP in a real-life clinical operative situation. In summary, these pilot data suggest that robotic RYGBP surgery results in less musculoskeletal stress to the upper back and possibly the upper extremities than standard laparoscopic technique. Robotic surgery also seems to offer both postural advantages and disadvantages, with more-ergonomic positioning in the upper and lower arm, wrist and wrist twist, but less-ergonomic positioning in the trunk. Current robotic workstations may induce greater neck strain from the monitor viewing angle. More-detailed studies are needed to fully assess the potential postural advantages of robotic surgical techniques over standard laparoscopy. Better understanding the ergonomics of laparoscopic and robotic surgery may lead to improved device and console design with the goal of minimizing musculoskeletal discomfort and reducing work-related injuries and fatigue.

\section{References}

1. Steinbrook R (2004) Surgery for severe obesity. N Engl J Med 350:1075-1079

2. Weber M, Muller MK, Bucher T et al (2004) Laparoscopic gastric bypass is superior to laparoscopic gastric banding for treatment of morbid obesity. Ann Surg 240:975-82; discussion $982-983$

3. Sjostrom CD, Lissner L, Wedel H, Sjostrom L (1999) Reduction in incidence of diabetes, hypertension and lipid disturbances after intentional weight loss induced by bariatric surgery: the SOS Intervention Study. Obes Res 7:477-484

4. Smith SC, Edwards CB, Goodman GN, Halversen RC, Simper SC (2004) Open vs laparoscopic Roux-en-Y gastric bypass: comparison of operative morbidity and mortality. Obes Surg 14:73-76

5. Berguer R (1998) Surgical technology and the ergonomics of laparoscopic instruments. Surg Endosc 12:458-462

6. Berguer R, Forkey DL, Smith WD (1999) Ergonomic problems associated with laparoscopic surgery. Surg Endosc 13:466-468

7. Nguyen NT, Ho HS, Smith WD et al (2001) An ergonomic evaluation of surgeons' axial skeletal and upper extremity 
movements during laparoscopic and open surgery. Am J Surg 182:720-724

8. Berguer R, Chen J, Smith WD (2003) A comparison of the physical effort required for laparoscopic and open surgical techniques. Arch Surg 138:967-970

9. Berguer R, Smith WD, Chung YH (2001) Performing laparoscopic surgery is significantly more stressful for the surgeon than open surgery. Surg Endosc 15:1204-1207

10. Berguer R, Rab GT, Abu-Ghaida H, Alarcon A, Chung J (1997) A comparison of surgeons' posture during laparoscopic and open surgical procedures. Surg Endosc 11:139-142

11. Patkin M, Isabel L (1995) Ergonomics, engineering and surgery of endosurgical dissection. J R Coll Surg Edinb 40:120 132

12. Hemal AK, Srinivas M, Charles AR (2001) Ergonomic problems associated with laparoscopy. J Endourol 15:499-503

13. Uhrich ML, Underwood RA, Standeven JW, Soper NJ, Engsberg JR (2002) Assessment of fatigue, monitor placement, and surgical experience during simulated laparoscopic surgery. Surg Endosc 16:635-639

14. Mohr CJ, Nadzam GS, Curet MJ (2005) Totally robotic Roux-en-Y gastric bypass. Arch Surg 140:779-786

15. Nio D, Bemelman WA, Boer KT, Dunker MS, Gouma DJ, Gulik TM (2002) Efficiency of manual versus robotical (Zeus) assisted laparoscopic surgery in the performance of standardized tasks. Surg Endosc 16:412-415

16. Talamini MA, Chapman S, Horgan S, Melvin WS (2003) A prospective analysis of 211 robotic-assisted surgical procedures. Surg Endosc 17:1521-1524

17. Lee EC, Rafiq A, Merrell R, Ackerman R, Dennerlein JT (2005) Ergonomics and human factors in endoscopic surgery: a comparison of manual vs telerobotic simulation systems. Surg Endosc 19:1064-1070

18. Ballantyne GH, Moll F (2003) The da Vinci telerobotic surgical system: the virtual operative field and telepresence surgery. Surg Clin North Am 83:1293-1304, vii
19. Ruurda JP, van Vroonhoven TJ, Broeders IA (2002) Robotassisted surgical systems: a new era in laparoscopic surgery. Ann R Coll Surg Engl 84:223-226

20. Ballantyne GH (2002) Robotic surgery, telerobotic surgery, telepresence, and telementoring. Review of early clinical results. Surg Endosc 16:1389-1402

21. Falk V, Diegler A, Walther T, Autschbach R, Mohr FW (2000) Developments in robotic cardiac surgery. Curr Opin Cardiol 15:378-387

22. Cadiere GB, Himpens J, Germay O et al (2001) Feasibility of robotic laparoscopic surgery: 146 cases. World J Surg 25:1467-1477

23. Yohannes P, Rotariu P, Pinto P, Smith AD, Lee BR (2002) Comparison of robotic versus laparoscopic skills: is there a difference in the learning curve? Urology 60:39-45; discussion 45

24. Ruurda JP, Wisselink W, Cuesta MA, Verhagen HJ, Broeders IA (2004) Robot-assisted versus standard videoscopic aortic replacement. A comparative study in pigs. Eur J Vasc Endovasc Surg 27:501-506

25. Ruurda JP, Visser PL, Broeders IA (2003) Analysis of procedure time in robot-assisted surgery: comparative study in laparoscopic cholecystectomy. Comput Aided Surg 8:24-29

26. Nio D, Bemelman WA, Busch OR, Vrouenraets BC, Gouma DJ (2004) Robot-assisted laparoscopic cholecystectomy versus conventional laparoscopic cholecystectomy: a comparative study. Surg Endosc 18:379-382

27. Dakin GF, Gagner M (2003) Comparison of laparoscopic skills performance between standard instruments and two surgical robotic systems. Surg Endosc 17:574-579

28. Ruurda JP, Broeders IA, Pulles B, Kappelhof FM, van der Werken C (2004) Manual robot assisted endoscopic suturing: time-action analysis in an experimental model. Surg Endosc 18:1249-1252 